\title{
Prácticas curriculares en la formación universitaria de los futuros profesionales: modelo para la actuación.
}

\section{Curricular internship in university training for future professionals: model for intervention.}

\author{
Carme Armengol Asparó \\ Universitat Autònoma de Barcelona \\ carme.armengol@uab.cat \\ Isabel Del Arco Bravo \\ Universitat de Lleida \\ del.arco@pip.udl.cat \\ Joaquín Gairín Sallán \\ Universitat Autònoma de Barcelona \\ joaquin.gairin@uab.cat \\ José Luís Muñoz Moreno \\ Universitat Autònoma de Barcelona \\ joseluis.munoz@uab.cat \\ David Rodríguez Gómez \\ Universitat Autònoma de Barcelona \\ david.rodriguez.gomez@uab.cat
} J. L. y Rodríguez Gómez, D. (2019). Prácticas curriculares en la formación universitaria de los futuros profesionales: modelo para la actuación. Revista Prácticum, 4(1), 19-36. 


\section{Resumen}

La aportación presenta algunos de los resultados más destacados que se derivan del estudio "Orientación y tutoría en las prácticas profesionales" que tiene por objetivos: analizar y delimitar la naturaleza y el sentido de las prácticas curriculares y concretar un modelo para la organización y el desarrollo de las prácticas curriculares. La metodología empleada ha considerado el análisis de documentos, la realización de 343 cuestionarios y 27 entrevistas a responsables institucionales, tutores académicos, tutores de los centros de prácticas y estudiantes. Los resultados evidencian de forma importante, y entre otras cuestiones, la necesidad de mejorar la coordinación entre la Universidad y los centros de prácticas alrededor del programa formativo de prácticas curriculares. De los resultados se deriva una propuesta de Modelo de Prácticas Curriculares que trata de focalizar el objetivo de intervención, los momentos, los protagonistas y el proceso. Las conclusiones destacan la necesidad de generar una mayor conciencia de la importancia personal, profesional e institucional que tienen las prácticas curriculares con el ánimo de estrechar los vínculos existentes entre la formación de los futuros profesionales y el ámbito del trabajo.

\section{Abstract}

This contribution presents some of the main results from a study of "Guidance and mentoring in professional practices" that had the following aims: to analyze and define the nature and meaning of curriculum practices and establish a model for the organization and development of curricular practices. The methodology has considered the analysis of documents, 343 questionnaires and 27 interviews with institutional leaders, academic tutors, professional tutors and students. Results show, among other things, the need to improve coordination between the university and "practice centers" around the curricular practice training program. From results, we derive a proposal for a "Curricular Practices Model" that focus on intervention aim, moments, agents and process. Conclusions highlight the need to generate greater awareness of the personal, professional and institutional importance of curricular practices, with the aim of strengthening the links between the training of future professionals and the workplace.

\section{Palabras claves}

Prácticas curriculares, Universidad, formación, profesionales.

\section{Keywords}

Curricular internship, University, training, professionals. 


\section{Introducción}

Entendemos las prácticas curriculares externas de carácter profesional como el conjunto de actividades organizadas en entornos laborales que realizan los estudiantes universitarios. Habitualmente, tienen una duración de meses y pretenden aplicar los conocimientos adquiridos a situaciones reales para la iniciación profesional.

La Universidad se enfrenta, cada vez más, a situaciones nuevas, distintas y complejas, como consecuencia de la realidad dinámica, de una sociedad exigente y de unos usuarios conscientes de la calidad de los servicios que precisan. En este escenario se requiere que las respuestas educativas consideren la complejidad de los fenómenos, las particularidades de cada contexto y las necesidades de los estudiantes, enfatizando en la importancia y necesidad de soportes de orientación para su óptimo desarrollo formativo.

En los últimos años la Universidad ha mostrado interés por orientar al estudiante, facilitando la transición desde la educación Secundaria Obligatoria (Gairín, Muñoz, Feixas y Guillamón, 2009 y Tortosa, Grau, Pellín, Roig, Pertegal, Sabroso, Ramírez y López, 2015), dándole soporte mientras cursa los estudios y realiza las prácticas curriculares y también apoyo en la transición hacia el mercado laboral. La orientación, planificada adecuadamente y con un seguimiento permanente es clave para que el estudiante alcance las competencias necesarias para adquirir una formación universitaria integral y de calidad (Zamorano, 2003). Ésta debe integrarse en la actuación docente dirigida al estudiante (Cano, 2008) dónde las prácticas curriculares adquieren un protagonismo necesario.

En la misma línea Camats, Flores, Del-Arco, Alaminos, Blázquez y Segura (2011) y Del-Arco, Camats, Flores, Alaminos y Blázquez (2011), defienden también esta finalidad orientadora en el momento de la elección de los estudios, en el momento de la matriculación y al configurar los itinerarios formativos, en el apoyo al estudio y al aprendizaje, orientación en el desarrollo de las prácticas curriculares, ayuda para la inserción laboral y profesional o para continuar la formación académica en estudios de postgrado o de tercer ciclo.

Algunas modalidades emergentes incorporan incluso, en el marco de la función tutorial, el desarrollo personal, humano y profesional del estudiante, tal y como acontece en Gran Bretaña con la "Planificación del Desarrollo Personal" y en Finlandia con los "Entornos de Aprendizaje Personalizados" (a partir de Montañés, Giménez, Blasco y Coll, 2008). Poco a poco se supera la concepción tradicional, y restringida, de vincular la tutoría tan sólo con la resolución de interrogantes sobre ciertas materias y contenidos (Cid, Pérez y Sarmiento, 2011). 
Los trabajos de Delaney (2004), Gallego (2004), Lim (2002) y Tinto (1975) sobre adaptación, integración, permanencia (Esteban, Bernardo y Rodríguez-Muñoz, 2016), retención y abandono de los estudiantes en la Universidad (Bernardo, Cerezo, Rodríguez-Muñoz y Tuerho, 2015), constatan la importancia de implementar actuaciones de orientación relacionadas con los distintos momentos de la vida del estudiante en la Universidad (promoción y acceso, acogida e incorporación, permanencia y egreso), para contribuir a un mejor desarrollo de los estudiantes a lo largo de su trayectoria académica en la universidad Universidad.

Si nos centramos en el tema objeto de estudio de la investigación que presentamos, la orientación centrada en las prácticas curriculares surge para dar apoyo y para superar aquellas dificultades que los estudiantes encuentran: en la toma de decisiones, la resolución de dilemas, adquiriendo hábitos de estudio y trabajo o en la inserción social y laboral (Bethencourt, Cabrera, Hernández, Álvarez y González, 2008 y González y Martín, 2004). La diversidad de actuaciones orientadoras dirigidas al estudiante tiene que caracterizarse a partir de sus propósitos, el carácter institucional y psicopedagógico que adoptan y el ámbito que abarcan e, inclusive, el momento en el que se sitúan. Especialmente porque estas prácticas suponen un entorno ideal para el desarrollo de competencias profesionales entre los estudiantes (Mareque y De Prada, 2018).

Además, la mejora de las prácticas curriculares como escenario formativo de los estudiantes universitarios exige, entre otros factores, de procesos bien organizados que minimicen las dificultades que tienen los estudiantes y de una óptima coordinación entre los diferentes profesionales e instituciones implicadas (Albertín y Villar, 2009). Requiere de una cultura institucional sostenida en el trabajo conjunto y la apertura al entorno, a partir del compromiso de la Universidad y de los centros de prácticas (Molina, 2007) por impulsar actuaciones de orientación que favorezcan el éxito de las prácticas curriculares en la formación inicial de los futuros profesionales. Desde esta óptica se revitalizan las aportaciones sobre orientación y tutoría (Gairín, Feixas, Muñoz y Guillamón, 2010 y Montero et al., 2014), los modelos de orientación universitaria (Álvarez y González, 2009) y la formación en alternancia (Rego, Rial y Barreira, 2015 y Tejada, 2012).

Las actuaciones debieran concebir la orientación como una actividad educativa, procesual e integrada en el programa formativo de prácticas y los planes de estudio. Supone otorgar una dimensión educativa a la orientación que, de acuerdo con Planas, Prieto y Lizandra (2011), implicaría educar para la vida, asesorar sobre opciones alternativas, aprender a tomar decisiones propias, generar un aprendizaje propio y guiar para reforzar, acompañar y dar soporte. Además, la calidad de los aprendizajes en este contexto 
puede mejorar a partir del compromiso que pueden asumir los involucrados en el proceso formativo (Ruiz, Sánchez y Mateu, 2018).

En este marco se ubica el estudio para la Mejora de la Calidad Docente desarrollado y que tiene como objetivo principal el buscar respuestas y alternativas a los problemas de filosofía, organización y seguimiento que plantea la realización de las prácticas curriculares en el contexto de los grados orquestados dentro del Espacio Europeo de Educación Superior. En concreto, los objetivos específicos del estudio han sido: analizar y delimitar la naturaleza y el sentido de las prácticas curriculares, reparando en su funcionamiento y problemáticas; y concretar un modelo para la organización y el desarrollo de las prácticas curriculares en la Universidad.

\section{Método}

La metodología llevada a cabo ha considerado el análisis de documentos, la realización de 343 cuestionarios y 27 entrevistas. En la aplicación de los cuestionarios participaron responsables institucionales $(11 \%)$, tutores académicos de la Universidad $(18,5 \%)$, tutores de los centros de prácticas $(17,6 \%)$ y estudiantes $(52,8 \%)$, pertenecientes a las áreas de ciencias sociales $(54,5 \%)$, humanas $(9,3 \%)$, salud $(29,9 \%)$ y experimentales y tecnológicas $(6,2 \%)$ de diversas universidades catalanas (Universidad Autónoma de Barcelona, Universidad de Barcelona, Universidad de Lleida, Universidad Politécnica de Catalunya, Universidad Ramón Llull, Universidad Abierta de Catalunya y Universidad Rovira i Virgili). En las entrevistas participaron 12 responsables institucionales, 6 tutores de los centros de prácticas, 2 coordinadores de prácticas y 7 estudiantes, también pertenecientes a las distintas áreas de conocimiento y universidades anteriormente mencionadas.

A la selección de la muestra para los cuestionarios siguió un procedimiento de muestreo por cuotas o accidental, para garantizar la presencia de las diversas categorías de agentes clave y áreas de conocimiento. En las entrevistas, el muestreo fue intencional a partir de la implicación de los participantes en las prácticas curriculares y su responsabilidad.

Las cuestiones consideradas en los cuestionarios, las entrevistas y el análisis de documentos quedaron agrupadas en cinco amplios bloques: datos de identificación (titulación, ámbito, tipología de participante, categoría profesional, género, edad y experiencia en programas de prácticas profesionales), organización del programa de prácticas (definición del modelo de prácticas profesionales, tipología de prácticas, soporte de las instituciones responsables, implicados y documentación entregada), contenido del programa de prácticas (competencias y objetivos, contenidos, recursos y sistema de evaluación), desarrollo (elección de centros, acogida en los centros y seguimiento de estudiantes) y mejora del programa de prácticas 
curriculares (coordinación interinstitucional, sistemas de feedback, sentido de la delimitación de indicadores, contenido de indicadores y uso).

De estos bloques y cuestiones se derivó la instrumentalización, que toma en cuenta los destinatarios y su naturaleza. Así, se concretó un cuestionario y un guión de entrevista, cuya elaboración responde a los siguientes pasos: a) selección de aspectos importantes; b) redacción provisional según destinatarios e introducción de datos de identificación; c) validación provisional por parte del equipo de investigación; d) aplicación en destinatarios para ajustar lenguaje y formato; y e) instrumentos definitivos.

\section{Resultados}

Los resultados de los cuestionarios evidencian que, en la organización de las prácticas curriculares, la implicación de la Universidad, por lo general, es adecuada. Un $80 \%$ de participantes valora positivamente la selección de los centros de prácticas, un 33\% considera que la información sobre las prácticas y la actividad profesional es poco adecuada y un $80 \%$ opinan que la implicación del centro de prácticas es bastante adecuada. Para el $93 \%$ el tutor del centro de prácticas desarrolla una función de dirección y asesoramiento al estudiante, pero un $87 \%$ considera que informa poco al estudiante sobre el sistema de trabajo y su implicación en las prácticas curriculares. Un $97 \%$ opina que las funciones que los estudiantes realizan con una mayor frecuencia son las de conocer y cumplir el proyecto formativo y colaborar con los profesionales del centro en lo que corresponda, siempre con una actitud de confidencialidad ante las informaciones del centro de prácticas. Sin embargo, el estudiante no suele comunicar a sus tutores las incidencias que afectan al desarrollo de las prácticas. Para el 92\%, siempre o a menudo se evalúa al estudiante de acuerdo a criterios establecidos y publicados. No es demasiado frecuente que el estudiante reciba información sobre prevención de riesgos laborales. Un $60 \%$ entiende que la documentación precisa se facilita con frecuencia, pero aquella vinculada al centro de prácticas (normativas, seguridad en el trabajo, etc.) no se hace con tanta frecuencia.

Del contenido del programa, cerca del $94 \%$ de participantes considera que las competencias que suelen desarrollarse con mayor frecuencia durante las prácticas tienen que ver con la observación y el análisis crítico que hace el estudiante en el contexto laboral, así como con su familiarización con las funciones y las tareas propias del entorno laboral. Tan sólo un 78,3\% entiende que se favorecen los valores de la innovación, la creatividad y el emprendimiento durante las prácticas. Con frecuencia, según el $81 \%$, se dan elementos suficientes al estudiante para poder realizar la memoria final de prácticas como recurso. Por el contrario, no existen demasiados 


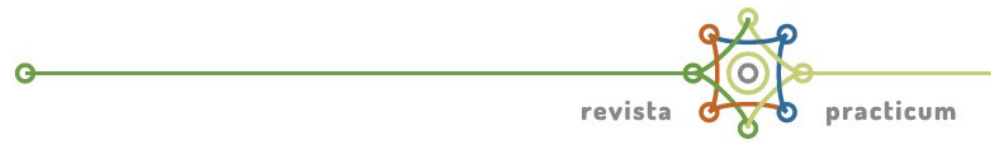

materiales de soporte al estudiante para realizar las prácticas en opinión del $65 \%$. Mayoritariamente, las directrices seguidas en la evaluación acontecen para el $80 \%$ y, particularmente, el $87 \%$ manifiesta que la evaluación contempla muy a menudo la capacidad personal demostrada (sentido de responsabilidad, facilidad de adaptación, iniciativa, implicación personal, etc.). No obstante, la retroalimentación y la devolución al estudiante sucede frecuentemente tan sólo para el 66,6\%.

En cuanto al desarrollo del programa, un $70 \%$ de los informantes cree que el estudiante selecciona el centro de prácticas por interés personal (conocer personas que trabajan en el centro de prácticas, proximidad a su lugar de residencia, perspectivas de empleabilidad futura, etc.). Se comprende así, por tanto, que el estudiante no escoge el centro de prácticas por el interés formativo que suscita su proyecto curricular. La acogida al estudiante en los centros de prácticas suele contener explicaciones de las tareas y las actividades a desarrollar, tal y como opina el $83 \%$. Sin embargo, no existe un plan de acogida en el $60 \%$ de los centros. El seguimiento establecido y dirigido al estudiante, así como las peticiones de los estudiantes para realizar reuniones donde tratar aspectos problemáticos, son útiles para el $83,7 \%$.

Y sobre los aspectos que podrían mejorar el programa, convendría insistir en la mejora de la comunicación y la coordinación entre tutores académicos y tutores de los centros de prácticas, puesto que suele darse en menos del $45 \%$ de las situaciones. Esto informa de la necesidad de trabajar aspectos diversos para mejorar la coordinación entre Universidad y centro de prácticas. De otro lado, los aspectos vinculados con el feedback presentan ocurrencias más homogéneas, siendo en más del $60 \%$ de los casos en la mayoría de los aspectos. En concreto, hay protocolos desarrollados sobre las prácticas según un $67,7 \%$; pero el análisis sistemático de los programas y la articulación de planes de mejora no se da con demasiada frecuencia ya que sólo ocurre en la realidad para el $56 \%$.

Las entrevistas llevadas a cabo posibilitan destacar como resultados los distintos puntos fuertes y débiles más habituales en cuanto al modelo de prácticas, la organización del programa, su contenido y desarrollo en los centros, tal y como señalamos en la Tabla 1. 
Prácticas curriculares en la formación universitaria de los futuros profesionales: modelo para la actuación.

\begin{tabular}{|c|c|c|}
\hline & Puntos fuertes. & Puntos débiles. \\
\hline $\begin{array}{l}\text { Modelo de } \\
\text { prácticas }\end{array}$ & $\begin{array}{l}\text { El aprendizaje del ejercicio } \\
\text { profesional. } \\
\text { La aplicación práctica. } \\
\text { La resolución de problemas. } \\
\text { El trabajo en equipo. } \\
\text { La conexión entre teoría y práctica. } \\
\text { La amplia oferta de centros de } \\
\text { prácticas. } \\
\text { La libertad de elección de centros. } \\
\text { La adecuada dedicación temporal. } \\
\end{array}$ & $\begin{array}{l}\text { La baja coordinación entre Universidad } \\
\text { y centros de prácticas. } \\
\text { La falta de reconocimiento que tienen } \\
\text { los centros de prácticas y sus tutores. } \\
\text { La falta de recursos y financiación. } \\
\text { El exceso de burocracia. } \\
\text { Las dificultades existentes para } \\
\text { gestionar convenios de colaboración. }\end{array}$ \\
\hline $\begin{array}{l}\text { Organización } \\
\text { del programa }\end{array}$ & $\begin{array}{l}\text { La predisposición de todas las } \\
\text { partes. } \\
\text { El soporte proporcionado. } \\
\text { El compromiso y la implicación de } \\
\text { las instituciones participantes. } \\
\text { La buena organización y gestión. } \\
\text { El control de la documentación. } \\
\text { Los protocolos de actuación. }\end{array}$ & $\begin{array}{l}\text { La poca consideración de las prácticas } \\
\text { en forma de créditos. } \\
\text { El excesivo número de estudiantes a } \\
\text { tutorizar. } \\
\text { El gran volumen de centros que se } \\
\text { deben gestionar. } \\
\text { La lentitud de los procesos } \\
\text { administrativos. } \\
\text { La falta de comunicación entre } \\
\text { tutores. } \\
\text { La poca supervisión durante las } \\
\text { prácticas. }\end{array}$ \\
\hline $\begin{array}{l}\text { Contenido del } \\
\text { programa }\end{array}$ & $\begin{array}{l}\text { La adecuación y pertinencia. } \\
\text { La especificidad y variedad según } \\
\text { temáticas y tipos de centros. } \\
\text { La orientación a la resolución de } \\
\text { problemas prácticos. } \\
\text { La conexión con la práctica } \\
\text { profesional. } \\
\text { La coherencia con las competencias } \\
\text { y los objetivos. } \\
\text { La dedicación de los tutores } \\
\text { académicos y de los centros de } \\
\text { prácticas. } \\
\text { La correcta definición de la } \\
\text { evaluación. }\end{array}$ & $\begin{array}{l}\text { La falta de concreción y conexión } \\
\text { entre teoría y práctica. } \\
\text { La ausencia de acompañamiento y } \\
\text { seguimiento. } \\
\text { La escasez de recursos y de } \\
\text { recompensas para los tutores de } \\
\text { centros de prácticas. } \\
\text { El poco tiempo disponible para la } \\
\text { tutorización. } \\
\text { La baja efectividad de la evaluación. } \\
\text { La no adopción de una perspectiva } \\
\text { multidimensional. }\end{array}$ \\
\hline $\begin{array}{l}\text { Desarrollo en } \\
\text { los centros }\end{array}$ & $\begin{array}{l}\text { El estudiante puede seleccionar } \\
\text { centro de prácticas. } \\
\text { La amplia y diversa oferta de } \\
\text { centros. } \\
\text { La implicación de los responsables } \\
\text { institucionales en la acogida y el } \\
\text { seguimiento continuado al } \\
\text { estudiante. } \\
\text { La colaboración entre tutores de la } \\
\text { Universidad y de los centros de } \\
\text { prácticas. }\end{array}$ & $\begin{array}{l}\text { La dispersión territorial de los centros } \\
\text { de prácticas. } \\
\text { El elevado número de peticiones } \\
\text { existentes para realizar las prácticas } \\
\text { en determinados centros. } \\
\text { La baja dedicación a la acogida al } \\
\text { estudiante en los centros de prácticas. } \\
\text { La escasa coordinación entre tutores } \\
\text { de la Universidad y de los centros de } \\
\text { prácticas. }\end{array}$ \\
\hline
\end{tabular}

Tabla 1, Puntos fuertes y débiles. Fuente: elaboración propia.

\section{Propuesta de modelo para la actuación}

La triangulación de las informaciones recabadas en el trabajo de campo mediante los cuestionarios, las entrevistas y también el análisis de documentos, permitió derivar en una propuesta de Modelo de Prácticas Curriculares (en adelante, MPC) centrado en la organización y el desarrollo generalizado de las prácticas curriculares. Los propósitos de este MPC se concretan en proporcionar pautas 


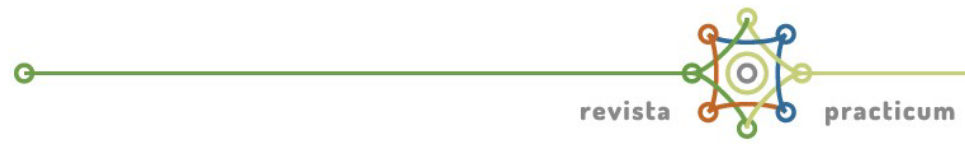

generales de actuación para la implantación y la aplicación de las prácticas curriculares universitarias, facilitar estrategias y herramientas que permitan la elaboración de propuestas relacionadas con la organización y el desarrollo de las prácticas curriculares universitarias y animar la actividad de los centros y a las titulaciones a sistematizar la realización de las prácticas curriculares universitarias.

La delimitación del MPC considera los elementos habituales en este tipo de propuesta: identificación de los elementos relevantes de la realidad analizada, descripción de los elementos relevantes, caracterización del funcionamiento del modelo y pautas para el seguimiento y la evaluación de su aplicación.

El estudio de diagnóstico hecho constata los elementos más característicos que intervienen en la delimitación y desarrollo de las prácticas curriculares universitarias. Su concreción queda sintetizada de acuerdo con el Figura 1.

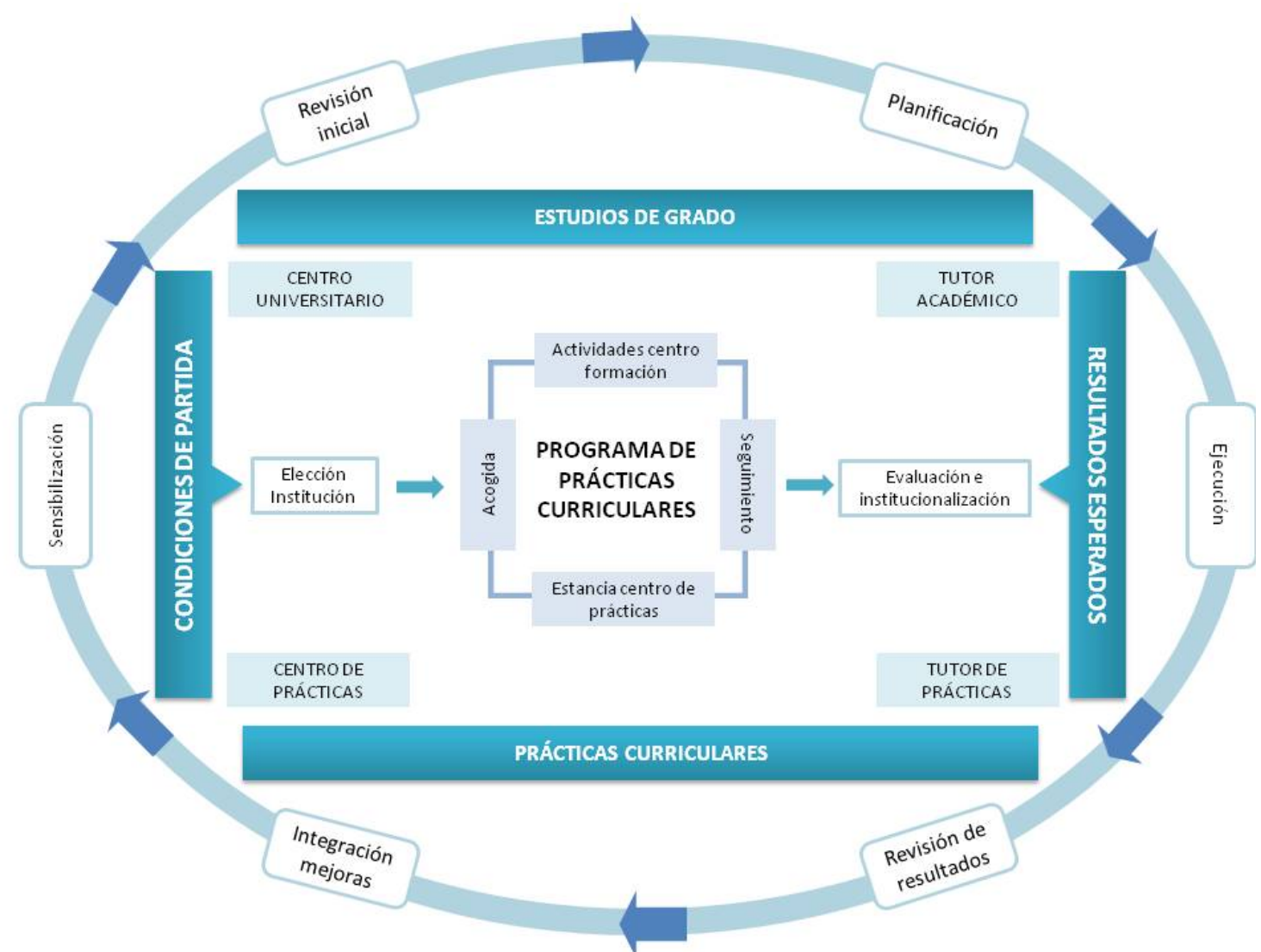

Figura 1, Modelo para la organización y desarrollo de las prácticas curriculares. Fuente: elaboración propia.

El MPC toma en cuenta cuatro aspectos fundamentales de los procesos de intervención y los elementos más significativos para cada uno de ellos. Particularmente, nos referimos al objetivo de intervención (¿qué?) es decir al programa formativo de prácticas curriculares, los momentos (¿cuándo?) en referencia a las situaciones clave en la aplicación del programa de prácticas curriculares, los 
protagonistas (¿quiénes?) personales e institucionales que participan en el desarrollo de la propuesta formativa y el proceso (¿cómo?) que debe garantizar una ejecución adecuada de la intervención formativa.

Los cuatros aspectos y los elementos vinculados interaccionan entre sí en un todo que busca la mejor coherencia interna y la adecuación a los programas de estudios y destinatarios considerados. La coherencia interna reside en el grado de congruencia existente entre todos los elementos puestos en juego. Por otra parte, las prácticas curriculares se encuadran dentro de un programa de Grado determinado y tienen que prestar atención a las finalidades y los objetivos del mismo. Además, el desarrollo de las prácticas queda condicionado por las condiciones de partida de los estudiantes y de los estudios en los que se matriculan.

Lo que, en última instancia, otorga sentido a la intervención es una ordenación que ayude a lograr la finalidad (¿para qué?) de los estudios. Por eso, la formación de los futuros profesionales será de calidad en la medida que todas las acciones formativas, incluyendo las prácticas curriculares, contribuyan a la consecución de las competencias y los objetivos formativos establecidos.

EL MPC se concibe como un "modelo para", en terminología pedagógica, por cuanto ofrece una secuencia para guiar la actuación y no solo una presentación de los elementos relevantes y más propia de los modelos descriptivos ("modelos de"). Además, puede relacionarse con un sistema de garantía de la calidad en tanto que trata, a través de un proceso organizado (momentos de sensibilización, revisión inicial, planificación, ejecución, revisión de resultados e integración de mejoras) y sujeto a controles, de garantizar la máxima calidad en la organización y el desarrollo de las prácticas curriculares.

Los distintos elementos considerados en los cuatro aspectos fundamentales en el MPC son los que sintetizamos en el Figura 2. Incluyen tanto cuestiones nucleares e imprescindibles como los procesos implicados en la dinámica organizativa que hacen posible su realización, y no dejan de ser cuestiones clave que debieran contemplarse en todo el diseño y desarrollo de las prácticas curriculares. 

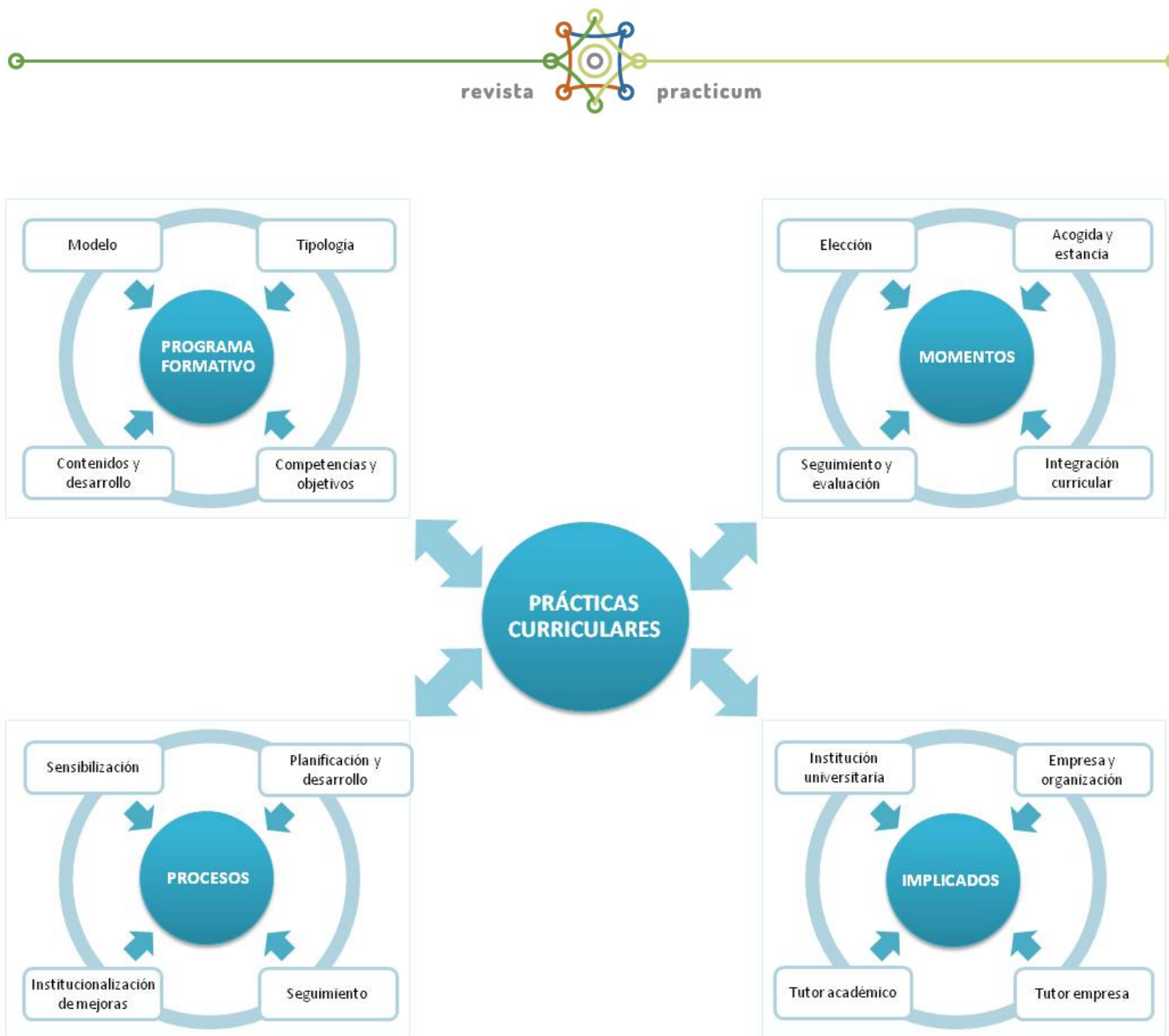

Figura 2: Componentes del Modelo de Prácticas Curriculares. Fuente: elaboración propia.

La esquematización posibilita, también, orientar procesos de análisis y mejora en relación al propio MPC. Así, y por ejemplo, se pueden investigar las relaciones existentes entre los distintos elementos y componentes fijados, establecer y verificar hipótesis y orientar la realización de informes de cumplimiento.

El éxito de las prácticas curriculares depende tanto de la calidad del diseño como del desarrollo que se haga del proceso. En este sentido, la caracterización de su funcionamiento e implantación puede articularse alrededor de las cinco siguientes fases.

- $\quad$ Fase de motivación, sensibilización y justificación.

Se trata de promover, mediante procesos de difusión, información y formación, actitudes favorables a la realización y aplicación del MPC. Seguramente, si se da a conocer el sentido y la utilidad del mismo, si se comprenden las ventajas y posibilidades que comporta en la formación de los estudiantes y si se alcanza la implicación de los diferentes agentes vinculados a su realización, habrá mayores garantías de éxito al mismo tiempo que, probablemente, podrían verse reducidas las resistencias al cambio que siempre existen. Esta fase puede culminar con un plan de trabajo y la puesta en marcha de una comisión responsable de la concreción del MPC. Asumir la importancia de las prácticas curriculares en todos los niveles de decisión de la institución universitaria, como 
instrumento necesario en la formación de los estudiantes, facilita su desarrollo y efectividad.

- Fase de planificación.

En esta fase se sistematiza el conjunto de actuaciones que configuran la propuesta que las instituciones consideran más adecuadas para la realidad en que se encuentran. Incluye tanto la detección de necesidades previas como la concreción de acciones y recursos implicados en función de los objetivos planteados. Además, se debe partir de las problemáticas planteadas y de los aspectos de mejora que la experiencia señala como necesarios. Partir de cuestionarios previos, entrevistas a informantes relevantes, información contrastada en grupos de discusión, lecturas críticas de informes de prácticas, etc., siempre contribuye a una detección real y no basada solamente en percepciones o suposiciones de los responsables institucionales. También puede ayudar en la implicación de estudiantes y profesorado en los procesos de mejora. Los objetivos de las prácticas curriculares son, desde esta perspectiva, la respuesta institucional frente a las necesidades y problemáticas planteadas. A partir de ellos se concretan los momentos de intervención, las actuaciones a realizar y sus responsables, los recursos que conviene utilizar y los mecanismos de evaluación correspondientes. Las concreciones pueden acompañarse de protocolos relativos a la organización del programa de prácticas (procesos implicados, responsabilidades de las instituciones y tutores, etc.), su desarrollo (sistema de elección de centros de prácticas, protocolos de acogida, sistema de seguimiento, etc.) y mejora (mecanismos de coordinación interinstitucional, sistemas de garantía de calidad, etc.).

- Fase de implementación.

Aquí es importante considerar la manera en cómo se materializan las actuaciones previstas, incluyendo los sistemas de información que se utilizan, los mecanismos de soporte al estudiante y la forma en cómo se identifican y resuelven los problemas puntuales presentados. Los mecanismos de seguimiento establecidos forman parte de esta base y bien utilizados pueden servir para la adaptación del MPC a situaciones concretas y a su mejor eficiencia.

- Fase de evaluación.

Los procesos de seguimiento no deben obviar la evaluación periódica de los resultados y sus efectos. De un lado, interesa verificar si los aprendizajes realizados por los estudiantes permiten desarrollar las competencias profesionales establecidas por la titulación para las prácticas curriculares. De otro lado, analizar los efectos que estas prácticas tienen sobre la formación integral de los estudiantes, sobre las relaciones interinstitucionales y sobre su capacidad para conseguir instalar nuevos conceptos de prácticas profesionales. Se trata, de algún modo, tanto de analizar los niveles de satisfacción de todos los implicados como de verificar los aprendizajes a los que sirven las prácticas curriculares y las 
trasformaciones que su realización promueve. También, de buscar y aplicar alternativas a situaciones poco satisfactorias.

- $\quad$ Fase de institucionalización.

La progresiva implantación del MPC y los procesos periódicos de seguimiento han de servir para reforzar y sedimentar las buenas prácticas. Es deseable que haya mecanismos para institucionalizar los procesos más efectivos, evitando la reconstrucción permanente de los mismos cada curso académico. La aprobación e institucionalización de protocolos e instrumentos que correspondan por los órganos de gestión competentes tiene que acompañarse de procesos de información y formación anuales a todos los implicados en las prácticas curriculares. Se trata así de socializar a los nuevos miembros en el funcionamiento institucional, a la vez que se saca provecho de los esfuerzos realizados y se refuerza la cultura institucional existente.

Con todo, los destinatarios directos del MPC son: Ios servicios universitarios responsables de las prácticas curriculares, los gestores universitarios de las prácticas curriculares (decanatos y coordinadores de titulación) y todo el personal relacionado directamente (estudiantes, tutores académicos, tutores de los centros de prácticas y personal de administración y servicios). El interés informativo, formativo e institucional del MPC puede ayudar a la formación práctica y efectiva de todos los estudiantes, a la sensibilización de la población universitaria sobre la necesidad y beneficios de las prácticas curriculares y a una consciencia de la importancia personal y profesional de vincular formación y trabajo.

Correspondería a las instituciones universitarias, las acciones vinculadas a la organización general de las prácticas curriculares y a la evaluación de sus resultados y efectos. Concretamente, podrían realizar acciones de sensibilización sobre la importancia de las prácticas curriculares, establecer las bases para el MPC de la Universidad, asesorar en la delimitación y concreción del MPC de las titulaciones, determinar el contenido general, formar a los gestores institucionales de las prácticas curriculares y evaluar los programas de prácticas.

Los gestores universitarios de las prácticas curriculares debieran establecer actuaciones relacionadas con el desarrollo y seguimiento de la propuesta formativa y particularmente: concretar con los implicados el MPC de las titulaciones, establecer y revisar periódicamente el mapa de instituciones colaboradoras de la Universidad, proponer anualmente los convenios de prácticas curriculares que correspondan, realizar la convocatoria anual para la petición y asignación de centros de prácticas, mantener contactos institucionales con las organizaciones no universitarias donde se realizan prácticas curriculares y en relación a su desarrollo, promover reuniones periódicas entre tutores de centros de prácticas y tutores universitarios, impulsar una formación propia para los tutores de prácticas curriculares, evaluar el desarrollo de las prácticas y sus 
efectos sobre la formación de los estudiantes y desarrollar un sistema de garantía de calidad en aquello que afecta a la implementación y mejora de las prácticas curriculares.

Los implicados directos en las prácticas curriculares se ocuparían de la realización, seguimiento y evaluación de las actividades dentro de las prácticas curriculares. Específicamente, podrían colaborar con los gestores en la concreción del MPC de la titulación, desarrollar las guías docentes de las prácticas curriculares en colaboración con los tutores de centros de prácticas, mantener contactos periódicos con los estudiantes en prácticas y con los tutores de centros de prácticas, informar al profesorado del curso correspondiente de los estudiantes que tutoriza y las actividades que se derivan de las prácticas, orientar a los estudiantes sobre los problemas que la práctica profesional plantea, realizar un seguimiento de las actividades de los estudiantes y evaluar sus progresos, identificar fortalezas y debilidades del sistema de prácticas curriculares y proponer mejoras y colaborar en la implantación y desarrollo del sistema de garantía de calidad en aquello que afecta a la mejora de las actividades relacionadas con las prácticas curriculares.

Los estudiantes, en esta relación, hacen realidad el contrato institucional de formación, aprovechando al máximo sus oportunidades formativas e implicación en las actividades de evaluación y mejora de las prácticas curriculares. Por su parte, el personal de administración y servicios de la Universidad facilita el desarrollo de las actividades formativas programadas.

\section{Conclusiones}

La organización y el desarrollo generalizado de prácticas curriculares exigen esquemas de trabajo que garanticen concreciones y resultados de acuerdo con los propósitos formativos de los estudios universitarios. Al respecto, el MPC propuesto y que se deriva del estudio realizado, trata de orientar su realización poniendo el énfasis en la orientación y también en la acción tutorial. Trata así de huir de propuestas prescriptivas y cerradas, proponiendo distintas herramientas posibles para facilitar que los diferentes estudios y universidades concreten su propia propuesta. En paralelo, se trata de favorecer el desarrollo de unas prácticas curriculares más vinculadas a unos escenarios y unas modalidades formativas próximas a los sistemas de calidad.

La propuesta de MPC procura despertar el interés informativo, formativo e institucional, en la dirección de ayudar a una mejor formación práctica y efectiva de los estudiantes como futuros profesionales, a la sensibilización de la población universitaria sobre la necesidad y beneficios de las prácticas curriculares y a generar una 


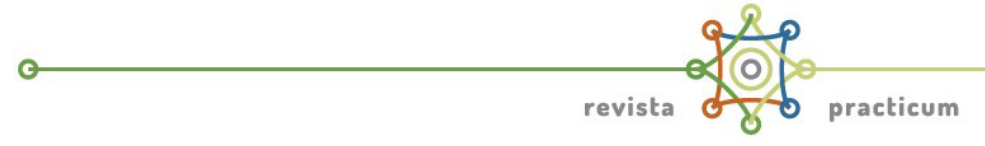

mayor conciencia de la importancia personal y profesional de vincular formación y trabajo.

Los tutores académicos de la Universidad y los profesionales de los centros de prácticas deben concretar su coordinación y sus funciones de acuerdo con el programa de prácticas establecido por la institución, considerando también las especificidades propias de los estudios y los centros de prácticas. En cualquier caso, el programa de trabajo debe especificar como mínimo las tareas que corresponden a los tutores, de la Universidad y de los centros de prácticas, en relación a la acogida, el seguimiento y la evaluación de los estudiantes; también, debería contemplar el grado de colaboración y trabajo conjunto entre tutores, así como su implicación en el programa de prácticas.

La realización efectiva de las prácticas curriculares implica a diferentes instancias y protagonistas, lo que exige de una práctica coordinada que responda a las lagunas normativas existentes, resuelva las problemáticas que la realidad plantea e intervenga como una estructura focalizada en el mejor servicio que se pueda dar desde la formación inicial de los estudiantes como futuros profesionales. Igualmente, su calidad queda en disposición de una buena planificación, un adecuado proceso de selección de instituciones colaboradoras y una valoración conjunta de todo el proceso (Hernández y Casillas, 2017). De hecho, las prácticas curriculares por sí mismas no son beneficiosas si no están debidamente planificadas (Silva, Del Arco y Flores, 2018).

Con esta propuesta de MPC se revitaliza la idea de que las acciones de orientación y tutoría (Gairín y otros, 2010) y de formación en alternancia (Tejada, 2012) acompañen a los procesos formativos, constituyéndose en aspectos básicos de la formación integral de los estudiantes universitarios. Se entiende de esta forma que orientar es ampliar el marco de experiencias, intereses, expectativas y oportunidades, promoviendo que los estudiantes, y futuros profesionales del mañana, desarrollen las competencias apropiadas para integrarse y sacar rendimiento de la formación universitaria y para su proyección a la integración social, laboral y profesional en el ámbito del trabajo.

La institucionalización del MPC, como proceso de construcción colectiva, a buen seguro exigirá tiempo y aprendizaje de la experiencia. Por eso, tan importante como los resultados es la realización de un proceso que consiga la participación y coordinación de todos los implicados. Un proceso de implantación del MPC debiera ser progresivo, lo que comporta delimitar un calendario de trabajo y responsables para llevarlo a cabo y, además, su mejora tiene que ser permanente y relacionarse con la identificación de buenas prácticas, la evaluación y la difusión de resultados para compartir el conocimiento y procurar que tanto las instituciones (universidades y centros de prácticas) como las personas crezcan y se desarrollen institucional y profesionalmente. 


\section{Referencias bibliográficas}

Albertín, P. y Villar, E. (2009). Valoración del prácticum. Aportaciones de los estudiantes en relación al prácticum de psicología de la Universidad de Girona. En UNIVEST 09. Girona: Universitat de Girona. Disponible en: https://bit.ly/2sMf7BQ

Álvarez, P. R. y González, M. C. (2009). Modelo comprensivo para la institucionalización de la orientación y la tutoría en la enseñanza universitaria. Revista Qurriculum, 22, 73-95. Disponible en: https://bit.ly/2MCYIP2

Behtencourt, J. T., Cabrera, L., Hernández, J. A., Álvarez, P. y González, M. (2008). Variables psicológicas y educativas en el abandono universitario. EJREP, 16, Vol. 6 (3), 603-622. Disponible en: https://bit.ly/2WfCi5j

Bernardo, A. B., Cerezo, R., Rodríguez-Muñoz, L. J. y Tuerho, E. (2015). Predicción del abandono universitario: variables explicativas y medidas de prevención. Fuentes: Revista de la Facultad de Ciencias de la Educación, Vol. 16, 63-84. Disponible en: https://bit.ly/2UhNH2M

Camats, R., Flores, O., Del-Arco, I., Alaminos, F., Blázquez, J. y Segura, J. (2011). Vers una cultura d'acollida a la universitat. En UNIVEST 11. Girona: Universitat de Girona. Disponible en: https://dugi-doc.udg.edu/handle/10256/3792

Cano, R. (2008). Modelo organizativo para la planificación y desarrollo de la tutoría universitaria en el marco del proceso de convergencia europea en Educación Superior. Revista Interuniversitaria de Formación del Profesorado, 22 (1), 185-206. Disponible en: https://bit.ly/2RS7N6G

Cid, A., Pérez, A. y Sarmiento, J. A. (2011). La tutoría en el prácticum. Revisión de la literatura. Revista de Educación, 354, 127154. Disponible en: https://bit.ly/2WlaxIw

Delaney, A. M. (2004). Ideas to enhance higher education's impact on graduate's lives: alumni recommendations. Tertiary Education and Management, 10, 89-105. Disponible en: https://bit.ly/2S5P3QK

Del-Arco, I., Camats, R., Flores, O., Alaminos, F. y Blázquez, J. (2011). An induction programme for Bologna first-year bachelor's degree students in Spain. Electronic Journal of Research in Educational Psychology, vol. 9 (1), 23, 445-462. Disponible en: https://eric.ed.gov/?id=EJ926505

Esteban, M., Bernardo, A. B. y Rodríguez-Muñoz, L. J. (2016). Permanencia en la Universidad: la importancia de un buen comienzo. Aula Abierta, Vol. 44, núm. 1, 1-6. Disponible en: https://bit.ly/2HzbF7V 


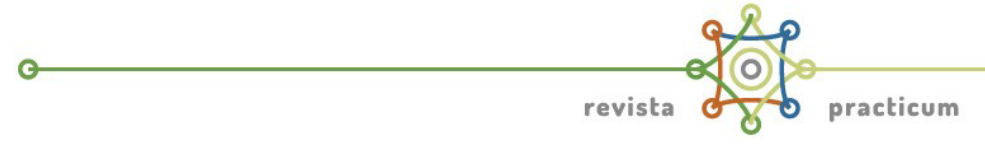

Gairín, J., Feixas, M., Muñoz, J. L. y Guillamón, C. (2010). La tutoría personalizada en la Universidad. Revista de Orientación Educacional, 45, 35-57.

Gairín, J., Muñoz, J. L., Feixas, M. y Guillamón, C. (2009). La transición Secundaria - Universidad y la incorporación a la Universidad. La acogida de los estudiantes de primer curso. Revista Española de Pedagogía, 42, 27-44. Disponible en: https://bit.ly/2sMrkqb

Gallego, Ma. I. (2004). Las tutorías personalizadas: una herramienta para facilitar la transición Secundaria - Universidad. En Actas XI Congreso Universitario de Innovación Educativa en las Enseñanzas Técnicas. Barcelona: UPC, 65-75.

González, I. y Martín, J. F. (2004). La orientación profesional en la Universidad. REOP, 15 (2), 229-315. Disponible en: https://bit.ly/2Ul8BxE

Hernández, J. P. y Casillas, S. (2017). Implementación y valoración de seminarios preparatorios para las prácticas externas en el Grado de Pedagogía. Revista Prácticum, 2(1), 40-59. Disponible en: https://bit.ly/2sMQaX4

Lim, H. (2002). Learner experience and achievement Project (LEAP). Survey Report 2002. Southampton Institute: www.Itsn.ac.uk

Mareque, M. y De Prada, E. (2018). Evaluación de las competencias profesionales a través de las prácticas externas: incidencia de la creatividad. Revista de Investigación Educativa, 36(1), 203-219. Disponible en: https://bit.ly/2S8YdvG

Molina, E. (2007). Evaluación de la formación: base para una propuesta de mejora del prácticum. Revista Evaluación e Investigación, Vol. 2 (2), 9-26. Disponible en: https://bit.ly/2G1yO0C

Montañés, E. Ma, Giménez, R., Blasco y O. Ma, Coll, V. (2008). La experiencia del prácticum en la Diplomatura en Turismo de la Universidad de Valencia: la inmersión del estudiante en el entorno laboral. En UNIVEST 08. Girona: Universitat de Girona. Disponible en: https://bit.ly/2FQXYQ7

Montero, Ma. J., Moya, M., López, L., García, Ma. E.; Hernández, J. y Villar, J. R. (2014). Propuestas de mejora para la orientación académica y profesional del alumnado: trinomio Universidad - alumno - empresa. La formación universitaria transversal y las realidades de mercado. Revista CIDUI 2014, 2, 114. Disponible en: https://bit.ly/2SdIb3u

Planas, J. A., Prieto, J. y Lizandra, R. (2011). El plan de acción tutorial, en Máster en Intervención y Asesoramiento Psicopedagógico para Profesionales. Barcelona: Universidad Autónoma de Barcelona (Documento policopiado).

Rego, L., Rial, A. y Barreria, E. (2015). La formación en alternancia en la Universidad y en los ciclos formativos: aportaciones desde dos investigaciones realizadas en Galicia. Educar, Vol. 51/2, 349-371. Disponible en: https://bit.ly/2Mz24wH 
Ruiz, P., Sánchez, L. y Materu, R. (2018). La innovación pedagógica de la mano de la investigación-acción para mejorar la calidad de las prácticas externas de los Grados de Maestro/a en Educación Primaria y Educación Infantil. Revista Electrónica Interuniversitaria de Formación del Profesorado, 21(1), 33-49. Disponible en: https://bit.ly/2UiUPM7

Silva, P., Del Arco, I., Flores, O. (2018). El desarrollo de competencias profesionales en la formación inicial de maestros a través de la formación dual. Profesorado, 22(1), 347-367. Disponible en: https://bit.ly/2S8EM6h

Tejada, J. (2012). La alternancia de contextos para la adquisición de competencias profesionales en escenarios complementarios de educación superior: marco y estrategia. Educación XXI, 15 (2), 17-40. Disponible en: https://bit.ly/2FO8Z4E

Tinto, V. (1975). Dropout from higher education: a theoretical synthesis of recent research. Review of Educational Research, 45, 89125. https://doi.org/10.3102/00346543045001089

Tortosa, Ma. T., Grau, S., Pellín, N., Roig, R., Pertegal, M. L., Sabroso, A., Ramírez, F. J. y López, A. (2015). La cuarta transición: estado de la cuestión en las dos orillas. En XII Jornadas de Redes de Investigación en Docencia Universitaria: nuevas estrategias organizativas y metodológicas en la formación universitaria para responder a la necesidad de adaptación y cambio. Alicante: Universidad de Alicante, 160-172. Disponible en: https://bit.ly/2SbC8N5

Zamorano, S. (2003). La tutoría en la formación de formadores. En Michavila, F. y García, J. (Eds). La tutoría y los nuevos modos de aprendizaje en la Universidad. Madrid: UPMUNESCO. 